\title{
Thermophysical Properties of Methane: Orthobaric Densities and Some Thermal Properties*
}

\author{
Robert D. Goodwin** \\ Institute for Basic Standards, National Bureau of Standards, Boulder, Colorado 80302
}

(June 30, 1970)

\begin{abstract}
For use in the computation of thermodynamic functions, analytical descriptions are given for the following properties: the orthobaric densities and saturation temperatures; the heats of vaporization; the specific heats of saturated liquid; and the thermodynamic functions for ideal gas states.
\end{abstract}

Key words: Heats of vaporization; methane; orthobaric densities; specific heats of the saturated liquid; thermofunctions for ideal gas states; thermophysical properties.

\section{List of Symbols}

Subscripts $c$ and $t$ refer to critical and liquid triple points.

Subscripts $g$ and $l$ refer to vapor and liquid in equilibrium.

$a, b, c$, constant coefficients

$A_{i} \quad$ constant coefficients

$\beta, \quad$ an exponent, 0.36

$C_{v}, \quad$ molal heat capacity at constant volume

$C_{p}, \quad$ molal heat capacity at constant pressure

$C_{\sigma}, \quad$ molal heat capacity of saturated liquid

$d, \quad$ molal density

$\Delta, \quad$ any relative deviation, $\left(y / y_{\text {calc }^{-1}}\right)$

$E$, the internal energy

$\epsilon, \quad$ exponent in various expressions

$H, \quad$ the enthalpy

$J, \quad$ the joule

$k, \quad$ conversion factor, $101.325 \mathrm{~J} / \mathrm{l}$-atm)

$l, \quad$ the liter, $1000 \mathrm{~cm}^{3}$

mol, $\quad 16.042$ grams of methane

$P, \quad$ pressure, $1 \mathrm{~atm}=101325 \mathrm{~N} / \mathrm{m}^{2}$

$\Phi(T), \quad\left(E^{0}-E_{0}^{0}\right) / \mathrm{RT}$, internal energy function

$Q_{r}, \quad$ heat absorbed in a reversible change of state

$R$, the gas constant, $8.3143 \mathrm{~J} /(\mathrm{mol}-\mathrm{K}),[26]$

$\rho, \quad d / d_{t}$, density reduced at $d_{t}$

$S, \quad$ the entropy

$\sigma, \quad d / d_{c}$, density reduced at $d_{c}$

$T, \quad$ temperature, K, IPTS (1968)

$T_{s}(\rho)$, liquid-vapor coexistence temperature

$\tau, \quad T / T_{c}$, temperature reduced at $T_{c}$

$u, \quad\left(T_{c}-T\right) /\left(T_{c}-T_{t}\right)$, normalized temperature, origin at $T_{c}$
*This work was conducted at NBS under sponsorship of The American Gas Association. **Cryogenics Division, Properties of Cryogenic Fluids Section, National Bureau of Stand${ }^{* *}$ Cryogenics Division, Properties of Cryoge
ards Laboratories, Boulder, Colorado 80302. $v, \quad 1 / d$, molal volume

$w, \quad\left(T_{c} / T-1\right) /\left(T_{c} / T_{t}-1\right)$, inverse normal temp., origin at $T_{c}$

$\omega, \quad \epsilon / x$, an argument in eq (8)

$x, \quad T / 400$, reduced temperature for eq $(8)$

$Y$, function of $C_{\sigma}$, defined on figure 5

$z, \quad(1-\tau)$, reduced temperature with origin at $T_{c}$.

The number of digits, given for coefficients in the empirical functions of this report, is chosen to yield calculated results having at least the same number of figures as exist in the data to be described.

\section{Introduction}

The current need for thermodynamic properties of methane was described in our preceding report [1]. ${ }^{1}$ The present report concludes our selection of published properties data for coexisting vapor and liquid, and includes also a description of the ideal gas thermofunctions. All of these properties are placed in analytical form directly useful for thermal computations.

Temperatures are on the IPTS (1968), as in [1], and we use the same fixed-point constants: $T_{t}=90.66 \mathrm{~K}$, $P_{t}=0.1151 \mathrm{~atm},\left(0.01166 \mathrm{MN} / \mathrm{m}^{2}\right), d_{t}=28.0536 \mathrm{~mol} / l$ (liquid); and $T_{c}=190.53 \mathrm{~K}, P_{c}=45.346 \mathrm{~atm},(4.5947$ $\left.\mathrm{MN} / \mathrm{m}^{2}\right), d_{c}=10.15 \mathrm{~mol} / l$.

\section{The Orthobaric Densities}

For the densities of coexisting vapor and liquid methane the preponderance of published data is on the liquid phase $[2,3,4,5,6,7,8]$. For the vapor phase, therefore, we have estimated data at densities

${ }^{1}$ Figures in brackets indicate the literature references at the end of this paper. 
up to $1 / 3$ of $d_{c}$ by use of the vapor pressure equation and the virial equation of state. We used the representation of second virial coefficients based on data of Sengers, Klein, and Gallagher, as well as the third virial coefficient, both as described in [1].

Analytical description of the vapor densities is given by our new form, constrained to qualitatively acceptable behavior near the critical point [9]. To facilitate selection of significant figures in the coefficients, we use variables $u$ and $w$ normalized to a maximum value of unity (at the triple point: see list of symbols). Vapor densities now are described by use of exponent $\beta=0.36$,

$$
\begin{aligned}
& \ln \left(\rho_{g} / \rho_{c}\right)=A_{1} \cdot w+A_{2} \cdot u^{\beta}+\sum_{i=3}^{8} A_{i} \cdot u^{i-2}, \\
& A_{1}=-27.42 \quad 792 \quad A_{5}=-10.44 \quad 279 \\
& A_{2}=-1.56 \quad 983 \quad A_{6}=23.44 \quad 911 \\
& A_{3}=10.16 \quad 428 \quad A_{7}=-18.38 \quad 419
\end{aligned}
$$

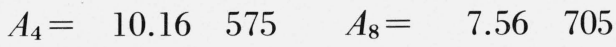

The value for exponent $\beta$ was selected by considering the published values, $\beta=0.367[10]$, and $\beta=0.3566$ [11]. The number of terms in (1) was selected to bring deviations down to the level of estimated uncertainty in data derived from the vapor pressure and virial equations. Individual relative deviations of vapor densities from eq (1) are plotted on figure 1. The rms deviation for 55 points is 0.21 percent.

Saturated liquid densities are described by our simple form [9] using argument $z \equiv(1-\tau)$,

$$
\begin{aligned}
& \rho_{l} / \rho_{c}-1=a \cdot z+b \cdot z^{\beta}+c \cdot \exp \left(-\epsilon \cdot \tau^{2} / z\right), \\
& a=0.62 \quad 253 \quad c=-0.08 \quad 367 \\
& b=1.89542 \quad \epsilon=0.6
\end{aligned}
$$

Inconsistent data were eliminated successively, leaving 60 points with an rms relative deviation of 0.24 percent. Figure 2 gives individual relative deviations from eq (2), and identifies the sources. At the triple point $90.66 \mathrm{~K}$, eq (2) gives a liquid density $d_{t}=28.0536$ $\mathrm{mol} / l$ resting primarily on the data of $[8]$, as seen on the figure.

Figure 3 gives the coexisting densities of vapor and liquid methane along the abcissa, calculated by eqs $(1,2)$. The ordinate gives the saturation temperature, $T_{s}(\rho)$. This plot provides a quick, rough estimate of the data. Conspicuously absent is the line for densities of freezing liquid. Some values recently have been estimated to $94.5 \mathrm{~K}$ (150 atm), including the volume change and heats of melting [12].

The rectilinear diameter may be derived by use of eqs $(1,2)$ for interpolation of $d_{g}$ and $d_{l}$ to the same temperatures. For 39 points at $112 \leqslant T \leqslant 188 \mathrm{~K}$, our representation in $\mathrm{mol} / l$,

$$
\begin{aligned}
\left(d_{g}+d_{l}\right) / 2 & =a+b \cdot z+c \cdot \exp (-\epsilon / z), \\
a=10.156, \quad b & =7.209, \quad c=0.183, \quad \epsilon=3 / 4,
\end{aligned}
$$

yields an rms deviation of 0.05 percent and a critical density $d_{c}=10.156+0.02 \mathrm{~mol} / l$, as compared with $d_{c}=10.15$ adopted for present work.

The relation, $T_{s}(\rho)$, is important in the nonanalytic equation of state [13]. For the combined data of figures 1 and 2 we obtain coefficients for the expression,

$$
\begin{aligned}
& T_{c} / T_{s}=1+|\sigma-1|^{3} \cdot F(\rho), \\
& F(\rho) \equiv A_{1} \cdot \ln (1 / \rho)+\sum_{i=2}^{9} A_{i} \cdot \rho^{i-2}, \\
& A_{1}=0.193073 \quad A_{6}=-112.50780 \\
& A_{2}=-0.34282 \quad A_{7}=141.02284 \\
& A_{3}=2.44483 \quad A_{8}=-89.60804 \\
& A_{4}=-12.24696 \quad A_{9}=\quad 22.74904 \\
& A_{5}=48.68975 \quad \mathrm{rms}=\quad 0.20 \%
\end{aligned}
$$

The signs and magnitudes of these coefficients are comparable with values found for other substances [9].

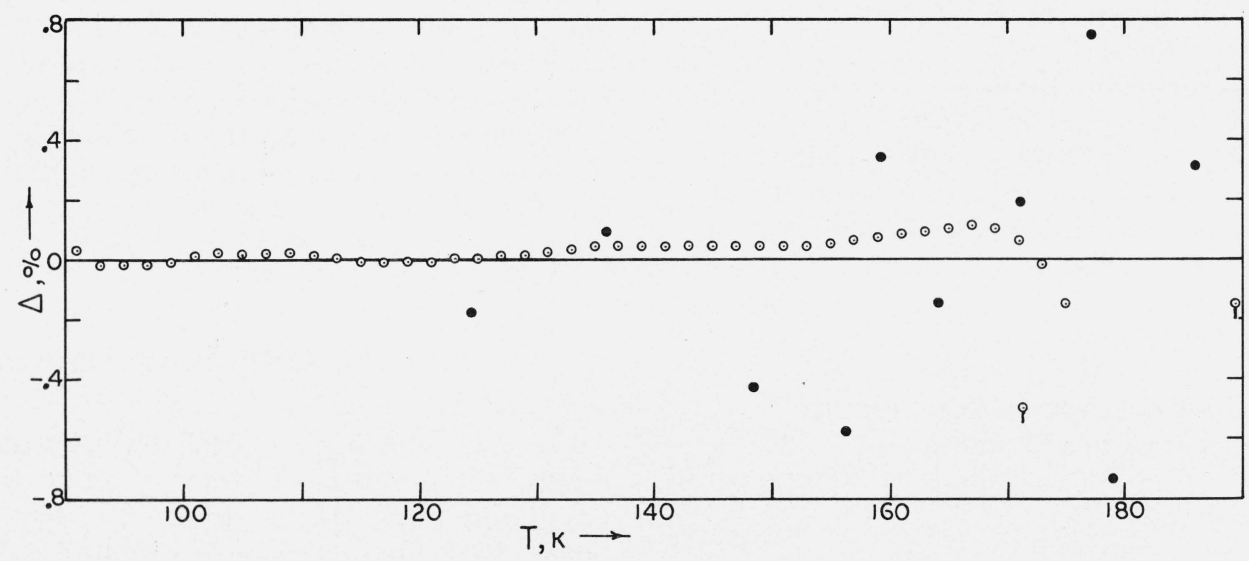

FIGURE 1. Relative deviations of the densities of saturated methane vapor from eq (1). 


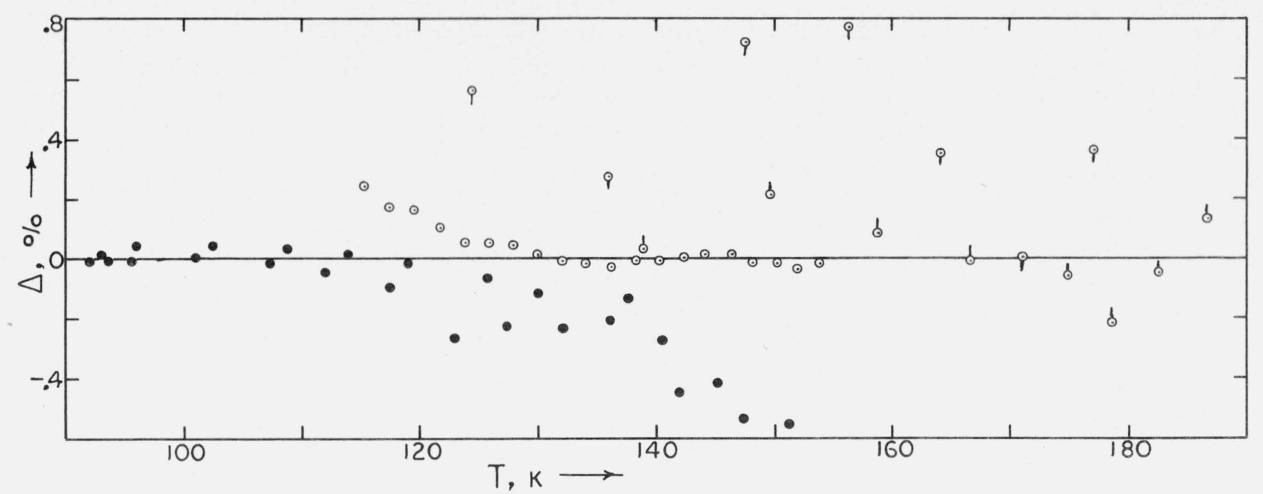

FIGURE 2. Relative deviations of the densities of saturated liquid methane from eq (2).

Filled circles from [8]; open circles from [7]; tails up from [5]; tails down from [4].

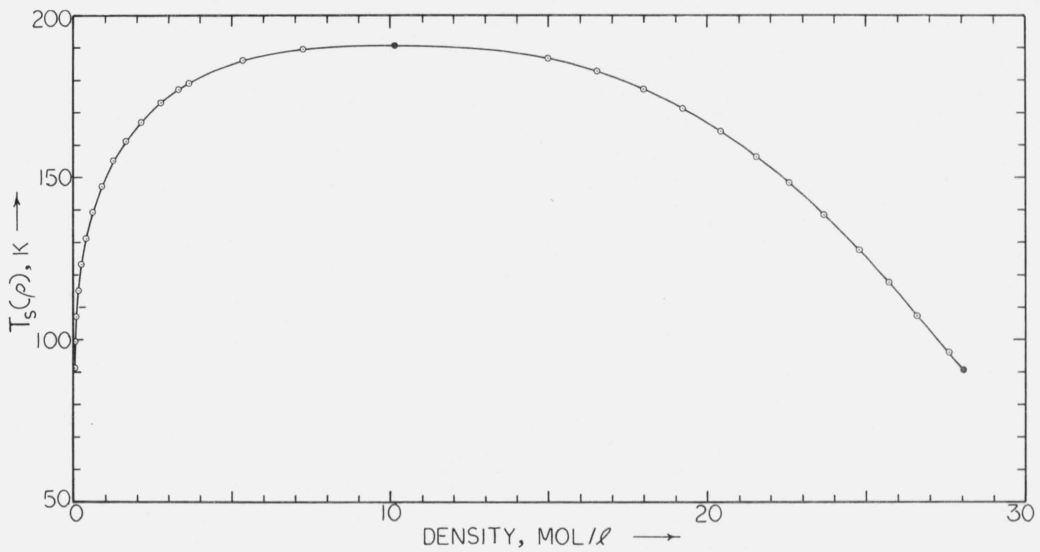

FiguRE 3. Methane saturation temperatures as a function of vapor and liquid densities up to the triple point.

\section{The Heats of Vaporization}

Some data for the heats of vaporization, $\Delta H_{v}$, have been reported [14, 15]. We now use the Clapeyron equation to calculate these heats by use of our vapor pressure equation [1] and eqs (1 and 2) of the present report,

$$
\Delta H_{v}=k \cdot T \cdot(d P / d T) \cdot\left(v_{g}-v_{l}\right) .
$$

Column 1 of table 1 gives the same rounded temperatures used in [14, 15]. (Experimental uncertainties do not demand conversion to the IPTS-1968.) Second and third columns of table 1 give the published data in kilo-Joules/mole. The fourth column gives our results via (5). The remaining three columns are the derived data used on the right of (5). Figure 4 gives the outline for our calculated results.

For some computations it would be convenient to use a more direct description of $\Delta H_{v}$ than given by (5). For the 21 points in the fourth column of table 1 the following representation gives a maximum relative deviation $\Delta=-0.04$ percent at $120 \mathrm{~K}$; deviations of 0.00 percent at $T=185$ and $T=190 \mathrm{~K}$; and an rms deviation of 0.02 percent,

$$
\begin{aligned}
& \Delta H_{v}=\sum_{i=1}^{6} A_{i} \cdot u^{i / 3}, \mathrm{~kJ} / \mathrm{mol}, \\
& A_{1}=7.88860 \quad A_{4}=-152.63520 \\
& A_{2}=-12.94022 \quad A_{5}=136.57252 \\
& A_{3}=76.33098 \quad A_{6}=-46.55421
\end{aligned}
$$

\section{Specific Heats of Saturated Liquid}

Specific heats of the liquid phase, along the coexistence path, are useful for computing thermodynamic properties into compressed liquid states. Data for $C_{\sigma}$ are derived from observations on the two phase, liquid-vapor system at constant volume by use of accurate PVT data for the two phases [17]. Published data for methane are quite uncertain because the authors failed to give either their experimental observations or a quantitative description of their derivations $[14,15]$. The data are shown by figure 5 in 
TABLE 1. Heats of vaporization for methane, $k J / m o l$

\begin{tabular}{|c|c|c|c|c|c|c|}
\hline \multirow{2}{*}{$T, K$} & \multicolumn{3}{|c|}{$\Delta H_{r}$} & \multirow{2}{*}{$\begin{array}{l}d P / d T \\
\mathrm{~atm} / \mathrm{K}\end{array}$} & \multirow{2}{*}{$\begin{array}{c}v_{y} \\
l / \mathrm{mol}\end{array}$} & \multirow{2}{*}{$\begin{array}{c}v_{1} \\
l / \mathrm{mol}\end{array}$} \\
\hline & [14] & [15] & Calc & & & \\
\hline 90.66 & & & 8.661 & 0.0147 & 64.1399 & 0.0356 \\
\hline 95.00 & & & 8.597 & .0227 & 39.4535 & .0361 \\
\hline 100.00 & 8.571 & 8.494 & 8.506 & .0353 & 23.8300 & .0367 \\
\hline 105.00 & 8.433 & 8.355 & 8.394 & .0522 & 15.1377 & .0373 \\
\hline 110.00 & 8.300 & 8.213 & 8.260 & .0741 & 10.0373 & .0379 \\
\hline 115.00 & 8.162 & 8.063 & 8.110 & .1014 & 6.9043 & .0385 \\
\hline 120.00 & 8.025 & 7.899 & 7.943 & .1344 & 4.9011 & .0392 \\
\hline 125.00 & 7.878 & 7.724 & 7.761 & .1734 & 3.5743 & .0400 \\
\hline 130.00 & 7.731 & 7.531 & 7.563 & .2186 & 2.6672 & .0408 \\
\hline 135.00 & 7.565 & 7.326 & 7.347 & .2702 & 2.0295 & .0416 \\
\hline 140.00 & 7.394 & 7.104 & 7.112 & .3283 & 1.5698 & .0425 \\
\hline 145.00 & 7.203 & 6.862 & 6.856 & .3930 & 1.2310 & .0436 \\
\hline 150.00 & 6.987 & 6.598 & 6.578 & .4645 & 0.9763 & .0447 \\
\hline 155.00 & 6.740 & 6.305 & 6.273 & .5431 & .7814 & .0460 \\
\hline 160.00 & 6.468 & 5.987 & 5.937 & .6292 & .6296 & .0475 \\
\hline 165.00 & 6.153 & 5.619 & 5.563 & .7232 & .5093 & .0493 \\
\hline 170.00 & 5.784 & 5.197 & 5.134 & .8262 & .4121 & .0514 \\
\hline 175.00 & 5.339 & 4.699 & 4.623 & .9397 & .3316 & .0542 \\
\hline 180.00 & 4.776 & 4.021 & 3.980 & 1.0664 & .2625 & .0579 \\
\hline 185.00 & 3.933 & 3.109 & 3.088 & 1.2122 & .1995 & .0636 \\
\hline 190.00 & & 0.782 & 1.267 & 1.4059 & .1269 & .0801 \\
\hline
\end{tabular}

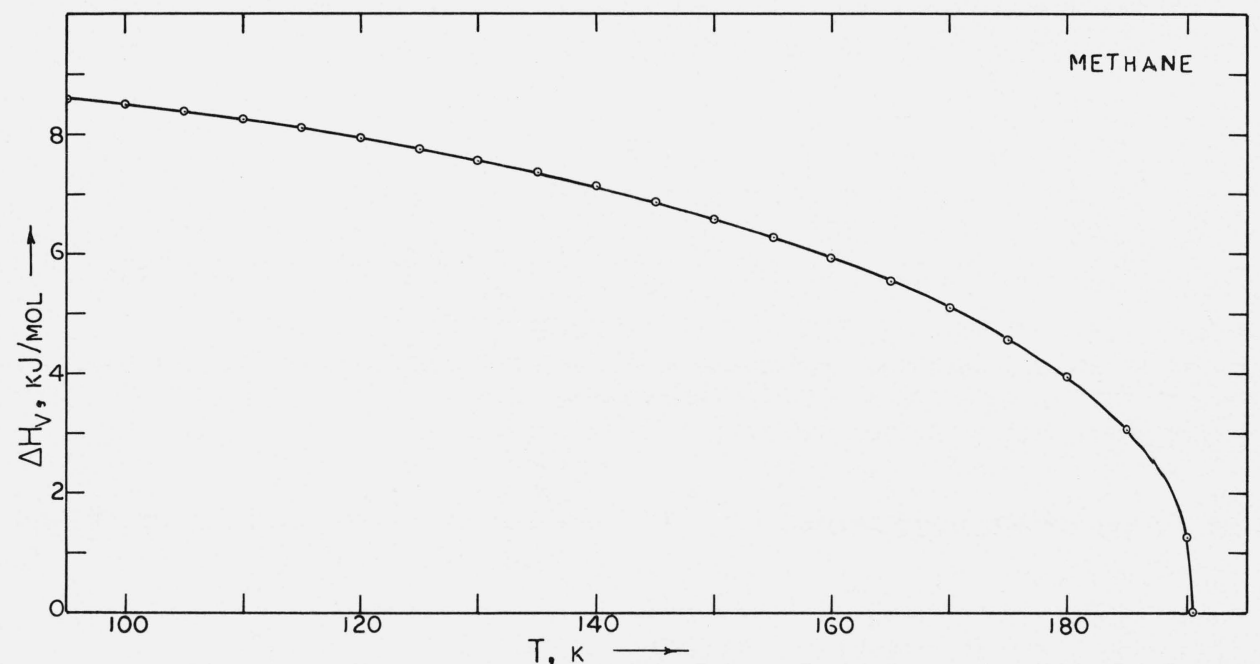

FIGURE 4. Outline for the heats of vaporization of methane, kilo-Joules/mol, derived via the Clapeyron equation with analytical descriptions of the physical properties.

coordinates $C_{\sigma} \cdot u^{\epsilon}$ versus $T$, the weighted form used for least squares determination of coefficients in

$$
\begin{aligned}
& C_{\sigma}=a+b \cdot u+c / u^{\epsilon}, \mathrm{J} /(\operatorname{mol~K}), \\
& a=29.250 \quad c=18.924 \\
& b=5.560 \quad \epsilon=0.55
\end{aligned}
$$

The value for exponent $\epsilon$ was selected by analogy: our oxygen data yield $\epsilon=0.54$ by trial, and our fluorine data give $\epsilon=0.59$ [18]. Present methane data yield $\epsilon=1.0 \pm 0.1$. The line on figure 5 is calculated from
(7). The rms relative deviation is 2.6 percent for 33 values of $C_{\sigma}$.

\section{The Ideal Gas States}

Thermodynamic functions for hypothetical ideal gas states at one atmosphere pressure may be derived for simple molecules as a function of temperature from spectroscopic data by statistical methods $[19,20]$. For our thermodynamic computations with PVT data, these ideal gas functions are invaluable because their 


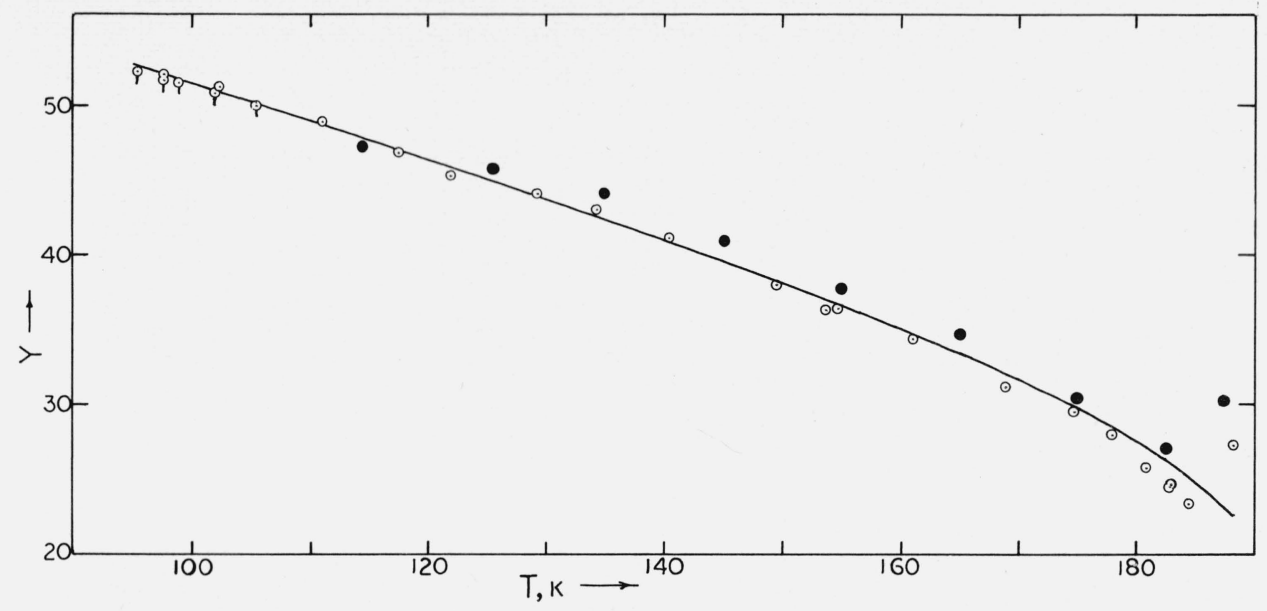

FIGURE 5. The function $\mathrm{Y} \equiv \mathrm{C}_{\mathrm{\sigma}} \cdot \mathrm{u}^{0.55}(\mathrm{~J} / \mathrm{mol}$ deg) for specific heat of liquid methane along the saturation path, using $\mathrm{u} \equiv\left(\mathrm{T}_{\mathrm{c}}-\mathrm{T}\right) /\left(\mathrm{T}_{\mathrm{c}}-\mathrm{T}_{\mathrm{t}}\right)$.

Filled circles are from [15]; open circles from [14]; circles with tails from [16]. The line is calculated with eq (7).

accuracy exceeds that which can be attained by experimental specific heat measurements.

Data for methane first were derived in API Project 44 [21], and work to 1961 has been reviewed [22]. For present work we select the recent results of McDowell and Kruse [23], and give them an analytical representation in the range $60 \leqslant T \leqslant 400 \mathrm{~K}$. With conventional notation [19], abbreviate the internal energy function by $\Phi(T) \equiv\left(E^{0}-E_{0}^{0}\right) / R T$, and define the argument $x \equiv T / 400$ with maximum value of about unity for our range of $T$.

A formulation with six constants was used for nitrogen [24]. For methane [23] and for oxygen [25] it does not give a highly precise representation of the specific heats. A more precise description is obtained with the power series,

$$
\Phi(T)=\sum_{i=1}^{10} A_{i} \cdot x^{i-1}
$$

On the other hand, a modification of the form given in [24] diminishes specific heat rms deviations by a factor of about $1 / 2$. Maximum deviations in $C_{v}^{0} / R$ (given below) become 0.02 percent in our present range of interest for methane, $80 \leqslant T \leqslant 360 \mathrm{~K}$. This accuracy is at least an order of magnitude better than can be obtained from PVT data when using the thermodynamic computation,

$$
C_{v}(\rho, T)=C_{v}^{0}(T)-T \cdot \int_{0}^{\rho}\left(\partial^{2} P / \partial T^{2}\right) \cdot d \rho / \rho^{2} .
$$

The modified form (8) uses an argument, $\omega \equiv \epsilon / x$. Constant $\epsilon$ is found by trial,

$\Phi(T)=A_{1}+A_{2} \cdot x^{1 / 3}+A_{3} \cdot x^{2 / 3}+A_{4} \cdot x+A_{5} \cdot \omega /\left(e^{\omega}-1\right)$,

$$
\begin{array}{rlrl}
\epsilon & =5.022880 & A_{3}=-1.847272 \\
A_{1} & =2.599898 & A_{4}=0.821122 \\
A_{2} & =1.444942 & A_{5} & =4.720791 .
\end{array}
$$

The specific heat is obtained via $C_{v}^{0}=d E^{0} / d T$,

$$
\begin{aligned}
C_{v}^{0} / R=A_{1}+\frac{4}{3} \cdot A_{2} \cdot x^{1 / 3}+\frac{5}{3} & \cdot A_{3} \cdot x^{2 / 3}+2 \cdot A_{4} \cdot x \\
& +A_{5} \cdot\left[\omega /\left(e^{\omega}-1\right)\right]^{2} \cdot e^{\omega} .
\end{aligned}
$$

The entropy is obtained via its definition,

$$
\Delta S \equiv Q_{r} / T=\int_{T_{1}}^{T}\left[C_{v} \cdot d T / T+P \cdot d v / T\right] .
$$

Introducing the published value of $S^{0} / R$ at $T_{1}=60 \mathrm{~K}$ yields,

$$
\begin{aligned}
S^{0} / R & =A_{0}+\ln (T / 60)+A_{1} \cdot \ln (x)+4 \cdot A_{2} \cdot x^{1 / 3} \\
& +\frac{5}{2} \cdot A_{3} \cdot x^{2 / 3}+2 \cdot A_{4} \cdot x+A_{5} \cdot\left[\omega /\left(e^{\omega}-1\right)-\ln \left(1-e^{-\omega}\right)\right]
\end{aligned}
$$

with constant $A_{0}=18.852484$.

Specific heat at constant pressure, and the enthalpy function now are simply

$$
\begin{aligned}
C_{p}^{0} / R & =1+C_{v}^{0} / R, \\
\left(H^{0}-E_{0}^{0}\right) / R T & =1+\Phi(T) .
\end{aligned}
$$

Table 2 gives temperatures in the first column. The next three pairs of columns give data and calculated values for $\Phi(T)$, for $C_{v}^{0} / R$, and for $S^{0} / R$.

Independent computations of above data by Harrison et al. [27] give almost identical results at $90 \leqslant T$ $<800 \mathrm{~K}$, further increasing confidence in their accuracy. 
TABLE 2. Ideal gas functions for methane at 1 atm

\begin{tabular}{r|c|c|c|c|c|c}
\hline \hline$T, K$ & $\Phi(T)$ & Calc & $C_{v}^{0} / R$ & Calc & $S^{0} / R$ & Calc \\
\hline 60.00 & 2.9693 & 2.9693 & 3.0014 & 3.0007 & 15.9337 & 15.9337 \\
80.00 & 2.9774 & 2.9774 & 3.0019 & 3.0021 & 17.0849 & 17.0849 \\
90.67 & 2.9803 & 2.9803 & 3.0021 & 3.0023 & 17.5859 & 17.5859 \\
100.00 & 2.9823 & 2.9823 & 3.0024 & 3.0023 & 17.9779 & 17.9779 \\
111.67 & 2.9844 & 2.9844 & 3.0027 & 3.0024 & 18.4197 & 18.4197 \\
120.00 & 2.9857 & 2.9857 & 3.0029 & 3.0026 & 18.7077 & 18.7077 \\
140.00 & 2.9882 & 2.9882 & 3.0042 & 3.0039 & 19.3248 & 19.3248 \\
160.00 & 2.9904 & 2.9903 & 3.0075 & 3.0075 & 19.8597 & 19.8596 \\
180.00 & 2.9927 & 2.9927 & 3.0153 & 3.0157 & 20.3321 & 20.3320 \\
200.00 & 2.9956 & 2.9957 & 3.0309 & 3.0313 & 20.7559 & 20.7559 \\
220.00 & 3.0000 & 3.0000 & 3.0571 & 3.0574 & 21.1412 & 21.1412 \\
240.00 & 3.0063 & 3.0063 & 3.0966 & 3.0966 & 21.4958 & 21.4958 \\
260.00 & 3.0152 & 3.0152 & 3.1506 & 3.1503 & 21.8257 & 21.8258 \\
273.15 & 3.0227 & 3.0228 & 3.1943 & 3.1939 & 22.0316 & 22.0316 \\
280.00 & 3.0272 & 3.0272 & 3.2196 & 3.2191 & 22.1357 & 22.1358 \\
298.15 & 3.0412 & 3.0412 & 3.2947 & 3.2942 & 22.4030 & 22.4030 \\
300.00 & 3.0428 & 3.0427 & 3.3030 & 3.3025 & 22.4296 & 22.4296 \\
320.00 & 3.0620 & 3.0619 & 3.3996 & 3.3994 & 22.7104 & 22.7103 \\
340.00 & 3.0850 & 3.0849 & 3.5077 & 3.5080 & 22.9803 & 22.9802 \\
360.00 & 3.1117 & 3.1117 & 3.6257 & 3.6264 & 23.2412 & 23.2412 \\
380.00 & 3.1420 & 3.1421 & 3.7516 & 3.7527 & 23.4947 & 23.4947 \\
400.00 & 3.1758 & 3.1759 & 3.8839 & 3.8847 & 23.7417 & 23.7418 \\
450.00 & 3.2737 & 3.2737 & 4.2328 & 4.2291 & 24.3369 & 24.3369 \\
\hline
\end{tabular}

\section{Discussion}

This report gives analytical descriptions of some methane properties, using existing and estimated data, as a base for preliminary computations of thermodynamic functions over the wide range from gaseous to compressed liquid states at low temperatures. It reveals deficiencies in the quantity of data available for densities of saturated vapor; in the precision of data available for saturated liquid densities; and hence uncertainty, difficult to assess, in the derived heats of vaporization. Data available for specific heats of liquid at coexistence cannot be assessed, due to a lack of published information. For thermofunctions in ideal gas states, the high quality of results derived by McDowell and Kruse suggests that they will stand for a long time.

This laboratory is undertaking an experimental program to obtain reliable and self-consistent measurements over a wide range of conditions and to compute thermodynamic properties based on their formulations.

\section{References}

[1] Goodwin, R. D., Thermophysical properties of methane: virial coefficients, vapor- and melting pressures, J. Res. Nat. Bur. Stand. (U.S.), 74A (Phys. and Chem.), No. 5, (Sept.-Oct. 1970).

[2] Keyes, F. G., Taylor, R. S., and Smith, L. B., The thermodynamic properties of methane, J. Math. \& Phys. 1, 211 (1922).

[3] Matthews, C. S., and Hurd, C. O., Thermodynamic properties of methane, Trans. Am. Inst. Chem. Engrs. 42, 55 (1946).

[4] Bloomer, O. T., and Parent, J. D., Liquid-vapor phase behavior of the methane-nitrogen system, Chem. Eng. Prog. Symposium Ser. 6, 49, 11 (1952).

[5] Vennix, Alan J., Low temperature volumetric properties and the development of an equation of state for methane, Thesis, Dept. of Chem. Engrn'g., Rice University, Houston, Texas, April, 1965.
[6] Grigor, Anthony F., The measurement and correlation of some physical properties of methane and per-deuteromethane, Thesis, Dept. of Chem., Pennsylvania State University, December, 1966

[7] Davenport, A. J., Rowlinson, J. S., and Saville, G., Solutions of three hydrocarbons in liquid methane, Trans. Faraday Soc. 62, 322 (1966).

[8] Terry, M. J., Lynch, J. T., Bunclark, M., Mansell, K. R., and Staveley, L. A. K., The densities of liquid argon, krypton, xenon, oxygen, nitrogen, carbon monoxide, methane, and carbon tetrafluoride along the orthobaric liquid curve, J. Chem. Thermodynamics 1, No. 4, 413 (1969).

[9] Goodwin, R. D., Estimation of critical constants $T_{c}, \rho_{c}$ from the $\rho(T)$ and $T(\rho)$ relations at coexistence, J. Res. Nat. Bur. Stand. (U.S.), 74A (Phys. and Chem.), No. 2, 221-227 (Mar.-Apr. 1970).

[10] Ricci, F. P., and Scafè, E., Orthobaric density of $\mathrm{CH}_{4}$ in the critical region, Physics Letters 29A, No. 11, 650 (1969).

[11] Jansoone, V., Gielen, H., DeBoelpaep, J., and Verbeke, O. B., The pressure-temperature-volume relationship of methane near the critical point, Physica 46, 213 (1970).

[12] Sindt, C. F., Ludtke, P. R., and Roder, H. M., Slush and boiling methane characterization, private communication 9758, this laboratory, July 1, 1970.

[13] Goodwin, R. D., Formulation of a nonanalytic equation of state for parahydrogen, J. Res. Nat. Bur. Stand. (U.S.), 73A (Phys. and Chem.), No. 6, 585-591 (Nov.-Dec. 1969).

[14] Wiebe, R., and Brevoort, M. J., The heat capacity of saturated liquid nitrogen and methane from the boiling point to the critical temperature, J. Am., Chem. Soc. 52, 622 (1930).

[15] Hestermans, P., and White, David, The vapor pressure, heat of vaporization and heat capacity of methane from the boiling point to the critical temperature, J. Phys. Chem. 65, No. 2, $362(1961)$.

[16] Clusius, Klaus, Uber die specifische Warme einiger kondensierter Gase zwischen 10 Grad abs. und ihrem triple Punkt, Z. Physik. Chem. (Leipzig) B3, 41-79 (1929).

[17] Goodwin, R. D., and Weber, L. A., Specific heats of oxygen at coexistence, J. Res. Nat. Bur. Stand. (U.S.), 73A (Phys. and Chem.), No. 1, 1-13 (Jan.-Feb. 1969).

[18] Goodwin, R. D., and Prydz, Rolf, Specific heats of fluorine at coexistence, J. Res. Nat. Bur. Stand. (U.S.), 74A (Phys. and Chem.), No. 4, 499-505 (July-Aug. 1970).

[19] Rossini, Frederick D., Chemical Thermodynamics (John Wiley \& Sons, Inc., New York, 1950).

[20] Mayer, J. E., and Mayer, M. G., Statistical Mechanics, (John Wiley \& Sons, Inc., New York, 1940). 
[21] Rossini, F. D., Selected values of physical and thermodynamic properties of hydrocarbons, Am. Petroleum Inst. Proj. 44, (Carnegie Press, Pittsburgh, Penna., 1953).

[22] See for example, Tester, H. E., METHANE, in Thermody. namic Functions of Gases, Vol. 3, Din, F., Editor (Butterworths, London, 1961).

[23] McDowell, R. S., and Kruse, F. H., Thermodynamic functions of methane, J. Chem. Eng. Data 8, 547 (1963). This was kindly pointed out by Lester Haar of NBS.

[24] Barieau, Robert E., Analytical expressions for the zero pressure thermodynamic properties of nitrogen gas including corrections for the latest values of the atomic constants and the new carbon-12 atomic weight scale, J. Phys. Chem. 69, 495 (1965).
[25] Stewart, R. B., The thermodynamic properties of oxygen, (Dissertation, Dept. of Mech. Engrn'g., Univ. of Iowa, June, 1966.)

[26] Mechtly, E. A., The international system of units (NASA SP-7012, Sept. 1966, U.S. Gov't. Printing Office, Washington, D.C. 20402.)

[27] Harrison, Roland H., private communication (U.S. Bureau of Mines Petroleum Research Center, Bartlesville, Oklahoma 74003, August, 1970.)

(Paper 75Al-645) 